$100,10, / 440$, should read $*, 0 \rightarrow 1,1,1,1,0,0$ and $0111<1110$, $0111<1101,0101<1001,11,100,10, / 444$, respectively. The expression " $n \geqq 4$," line 7 , page 89 , should read " $10>n \geqq 4$." In line 15 , page 100 , the symbol $[n /]$ should be $[n / p]$. On page 194 , line 2 , the phrase "modulo $p^{a-1}$ " should follow "is congruent." On page 299 the symbol $\left(p_{1} q\right)$ in the 9 th line from the bottom should read $\left(p_{1} / q\right)$, and $\left[\left(p_{k}-1\right) / 2\right]$ in line 3 from the bottom should read [ $\left.\left(p_{i}-1\right) / 2\right]$.

On the whole the authors have succeeded in presenting number theory in a fascinating and highly instructive manner. Their work will fill an important place in the literature.

\title{
Rufus Oldenburger
}

Differential Geometrien in den Kugelräumen. Vol. 1. Konforme Differentialgeometrie von Liouville und Möbius. By T. Takasu. Tokyo, Maruzen, 1938. $18+457 \mathrm{pp}$.

This handsome textbook gives a comprehensive account of the differential geometries in spaces in which spheres are taken as spatial element. It is the outgrowth of a series of fundamental investigations in which the author has been engaged since 1924, and which have, so far, been laid down in 47 papers. He has also used, in the widest and most generous sense of the word, the work of other authors, which he lists in an extensive and probably complete bibliography of 202 titles. This book is, therefore, the most authoritative source on the subject, and the reader will at the same time be pleased with the variety and beauty of the results and amazed at the thoroughness of the work.

The main body of research already has been published in various "Science Reports of the Tôhoku Imperial University," beginning in March, 1928. The present volume of ten follows the previous publications, but also departs from it, giving more material and more references. The author, in the preface, gives his own classification of the different geometries as derived from Lie's geometry of the spheres, embracing several types of conformal geometry, including that of Laguerre, of affine, and of non-euclidean geometry. We repeat his fundamental principle as follows:

"1. The conformal space of Möbius is a non-euclidean space with movable absolute spheres, hence also with variable curvature.

"2. The space of Laguerre is a euclidean space with movable absolute circles, hence also with variable unit of angular measurement.

"3. The space of Lie is a conformal space with a movable absolute complex of spheres and at the same time a dual-conformal space 
with movable dual-absolute complex of spheres, so that the unity of measure of length and angle are functions of position."

These three principles, according to the author, allow a perfectly clear interpretation of the analytical construction of these geometries, and one which has no gaps. He believes that this elucidates completely a field which has had too little attention.

The geometry of spheres has, indeed, been little investigated and mostly in an unsystematic way. Pentaspherical and hexaspherical coordinates, which are the appropriate types of coordinates for such geometries, were originally only used by Darboux (1889) and Coolidge (1916), until after 1922 when a new series of investigations began, with which the names of Vessiot, Thomsen, Blaschke, Liebmann and Kubota are connected, of whom some also use cartesian coordinates. There is a close relation in method between this book of Professor Takasu and the third volume of Blaschke's Differentialgeometrie, written by Thomsen, and which appeared in 1928. There is also a considerable difference between the books, Thomsen's book being only partly devoted to differential geometry, Takasu investigating in a very systematic way only differential geometry.

This book of 457 pages is only the first volume of a planned set of three. It deals with the geometries of Liouville and Möbius. The second volume will give the differential geometry of Laguerre, the third volume that of Lie.

The book consists of two "Abschnitte," a short first one of twentyfive pages giving introductory theories, explaining the relation of the three geometries of the sphere, the second one bringing the theory of systems of $\infty^{1}$ circles, of nets of curves, of systems of $\infty^{1}$ and $\infty^{2}$ spheres, of systems of $\infty^{1}$ circles in space, and of triply orthogonal systems. It follows closely the classical exposition of ordinary differential geometry. The systems of $\infty^{1}$ circles correspond to the curves in the plane (taken as envelopes of straight lines), the systems of $\infty^{1}$ spheres to curves in space, the systems of $\infty^{2}$ spheres to surfaces, the systems of $\infty^{1}$ circles in space to ruled surfaces, the correspondence is with non-euclidean geometry rather than with euclidean because of the nondegenerated quadratic relations between the coordinates.

It is impossible to mention even a small part of the rich material. We have generalizations to conformal space of Bertrand curves, of the four vertex theorem, of Meusnier's and Euler's theorems. Many classical results receive for the first time their proper setting. Dualities are often shown by comparison of theorems and proofs on the right and left side of the page. The systematic exposition can often reframe an old proof with the new and unified methods in the new 
conformal language and notation. There is a certain finality about the content which makes it the foundation of every further study on the subject.

It is not always easy to follow the author and we would like to ask him to be kind with his readers when he gives the final touches to the other two volumes. As an example, let us take the beginning of the book. On page 1 we hurry immediately into the midst of things. An oriented sphere is defined by 5 pentaspherical coordinates connected by a quadratic relation. The meaning of these coordinates is not explained, we must take this from other books, and the quadratic relation is only given as $(y y)_{5}=1$, which leaves it to the reader to discover what it means. We are not informed either, whether the coordinates are ordinary or general pentaspherical coordinates, and have to discover this later, from the context. On page 2 we read that the different projective orientation processes must be understood in the sense of the author's non-euclidean geometry. This is an essential point, and we must therefore first go to volume 26 of the Tôhoku Mathematical Journal (1926) to find what it means. The same thing happens on page 3 , where we are referred to another paper to find the meaning of certain equations expressing a doubly oriented sphere. Such difficulties could easily be avoided if the author, at the beginning, would not presuppose more than an average college knowledge of pentaspherical coordinates, projective and non-euclidean geometry, as, for example, Blaschke, Thomsen, or Fubini-Cech have done in their related expositions. We are sure that Professor Takasu will only do justice to his beautiful investigations if he can agree to such modifications in his presentation.

D. J. Struik

Superficie Razionali. By Fabio Conforto. Bologna, Zanichelli, 1939. $16+549$ pp.

Although there is an extensive literature on rational surfaces, it is scattered through the periodicals in various languages, and the methods of proof differ widely as the theory gradually develops through more than a century. On the other hand, a knowledge of this field is indispensable to the study of algebraic geometry of more than two dimensions, and to some phases of analysis.

The purpose of the present book is to supply this need of a systematic development of the subject from the present point of view, starting at the beginning and providing all the necessary details of the general theory, but referring to original papers for further 